\section{SOCCER PLAYERS WHO RUPTURE THEIR ACL DEMONSTRATE BIOMECHANICAL RISK FACTORS WHILE DECELERATING: A PROSPECTIVE COHORT STUDY}

${ }^{1}$ Celeste Dix, ${ }^{2}$ Amelia Arundale, ${ }^{3,4}$ Holly Silvers-Granelli, ${ }^{5}$ Ryan Zarzycki, ${ }^{6}$ Adam Marmon, 1,7 Lynn Snyder-Mackler. 'University of Delaware Biomechanics and Movement Science Program, Newark, USA; ${ }^{2}$ Mount Sinai Hospital, New York, USA; ${ }^{3}$ Director of Research Major League Soccer Medical Assessment Research Committee (M-MARC), New York, USA; ${ }^{4}$ Velocity Physical Therapy, Los Angeles, USA; ${ }^{5}$ Arcadia University Department of Physical Therapy, Glenside, USA; ${ }^{6}$ LiteCure LLC., Newcastle, USA; ${ }^{7}$ University of Delaware Department of Physical Therapy, Newark, USA

\subsection{6/bjsports-2021-IOC.45}

Background Many non-contact anterior cruciate ligament (ACL) injuries amongst women's soccer players occur during a deceleration manoeuvre. Retrospective video analysis of ACL injuries has demonstrated that players are often in knee valgus at the time of injury. Whether the components of valgus collapse: hip adduction, hip internal rotation, and knee abduction angles during a deceleration task, differ between players who go on to ACL injury has not been prospectively examined. In order to develop more effective injury prevention programs, it is necessary to understand these risk factors in the context of a sport-specific task.

Objective To compare whether hip and knee kinematics during a deceleration differ between collegiate women's soccer players who go on to sustain ACL injury and those who don't.

Design Prospective cohort non-randomized intervention study. Setting Collegiate women's soccer.

Patients (or Participants) 51 collegiate women's soccer players. Interventions (or Assessment of Risk Factors) Preseason, threedimensional motion analysis of a deceleration.

Main Outcome Measurements Descriptive statistics for injured limbs and the mean of uninjured players' limbs were calculated for peak values of valgus collapse components (hip adduction, hip internal rotation, knee abduction). A MANOVA was also used to determine whether there were biomechanical differences between Injured $(\mathrm{N}=4)$ and Uninjured $(\mathrm{N}=47)$ players.

Results There was a significant difference between groups for hip adduction angle $(\mathrm{p}=0.03)$. The Injured players were in much more hip adduction $\left(8.63 \pm 4.1^{\circ}\right)$ than those who remained Uninjured $\left(1.66 \pm 6.0^{\circ}\right)$. There were no other significant or clinically meaningful differences between groups for hip internal rotation or knee abduction angle.

Conclusions Hip adduction during deceleration differed between players who went on to ACL injury and those who did not. Therefore, the components of knee injury prevention programs that address lateral and posterior hip strength and neuromuscular control are likely crucial components of ACL injury prevention programing for soccer players.

\section{ABSTRACT WITHDRAWN}

\section{AGE-APPROPRIATENESS OF COMMON NEUROMUSCULAR TRAINING EXERCISES IN ACL INJURY PREVENTION PROGRAMS}

Daphne Ling, Caroline Boyle, Joseph Janosky, Brenda Chang, Naomi Roselaar, James Kinderknecht, Robert Marx. Hospital for Special Surgery, New York, USA
Background The appropriateness of neuromuscular training exercises across different age groups has not yet been investigated, particularly in younger children.

Objective This study will determine which neuromuscular training exercises can be performed with proper alignment in various age groups.

Design Cross-sectional study.

Setting Youth athletes in a controlled setting.

Participants Children ranging from 8-17 years of age were recruited from schools and youth sports organizations. A total of 360 participants were evaluated (8-11 years: 165, 54\% female; $12-15$ years: $136,40 \%$ female, $16-17$ years: 59, 53\% female).

Interventions Seven exercises were selected for evaluation. Participants completed two trials of each exercise and were judged on maintaining neutral body alignment after receiving visual/verbal instruction on the first trial and feedback cues on the second trial.

Main Outcome Measurements Three evaluators judged each exercise, which was deemed as correct when at least two evaluators agreed that neutral alignment was maintained. Comparisons were made across ages and between sex using the Chisquared test or Fisher's exact test. The proportions of participants who performed the exercise correctly were also compared before and after feedback cues were provided.

Results There were no significant differences in performance across ages and sex for nearly all exercises. Most neuromuscular training exercises had a less than 50\% success rate for proper neutral alignment in children $8-17$ years of age. The use of visual and verbal feedback cues significantly increased the proportion of participants who correctly completed the exercise $(p<0.001)$. Inter-rater reliability among the evaluators ranged from kappa $=0.31$ to 0.42 , showing fair to moderate agreement.

Conclusions These results demonstrate the importance of training coaches and physical education teachers to provide cues that reinforce proper technique during ACL injury prevention exercises. Children should perform common neuromuscular training exercises with feedback on proper technique. The availability of age-appropriate exercises may help increase adherence to injury prevention programs and maximize their effect on reducing injuries.

\section{DO NON-ELITE SOCCER TEAMS IMPLEMENT NEUROMUSCULAR TRAINING TO PREVENT NON- CONTACT ANTERIOR CRUCIATE LIGAMENT INJURIES?}

Christophe Eechaute, Lieselot Longé, Winnie Debecker, Lore Meganck, Lynn Leemans, Ronald Buyl, David Beckwée, Tim Hendrikx, Michel Stéphanie. Vrije Universiteit Brussel, Brussels, Belgium

\subsection{6/bjsports-2021-IOC.47}

Background Soccer is one of the highest risk sports for anterior cruciate ligament (ACL) injuries. These injuries have serious long-term consequences and cause substantial socio-economic costs. The preventive effect of an exercise program is highly dependent on its components and the level of compliance. In Belgium, it is unknown whether non-elite soccer teams implement neuromuscular training to prevent ACL injuries.

Objective Is preventive neuromuscular training incorporated during practice in non-elite Belgian soccer?

Design Prospective cohort study.

Setting Non-elite soccer teams. 